\title{
Comparison of short-term outcomes between single-incision plus one-port laparoscopic surgery and conventional laparoscopic surgery for distal gastric cancer: a randomized controlled trial
}

\author{
Wenhao Teng ${ }^{1}$, Jingfu Liu ${ }^{2}$, Wenju Liu ${ }^{1}$, Jianping Jiang', Meimei Chen ${ }^{1}$, Cheng Wei ${ }^{1}$, Choon Seng Chong ${ }^{3}$, \\ Weidong Zang ${ }^{1}$ \\ ${ }^{1}$ Department of Gastrointestinal Surgery, Fujian Medical University Cancer Hospital, Fujian Cancer Hospital, Fuzhou, China; ${ }^{2}$ Department of \\ Blood Transfusion, Fujian Medical University Cancer Hospital, Fujian Cancer Hospital, Fuzhou, China; ${ }^{3}$ Division of Colorectal Surgery, University \\ Surgical Cluster, National University Hospital, Singapore, Singapore \\ Contributions: (I) Conception and design: W Teng, W Zang; (II) Administrative support: W Zang; (III) Provision of study materials or patients: W \\ Teng, W Liu, J Jiang; (IV) Collection and assembly of data: W Teng, M Chen, C Wei; (V) Data analysis and interpretation: W Teng, J Liu, W Zang; \\ (VI) Manuscript writing: All authors; (VII) Final approval of manuscript: All authors. \\ Correspondence to: Weidong Zang. Department of Gastrointestinal Surgery, Fujian Medical University Cancer Hospital, Fujian Cancer Hospital, \\ Fuzhou 350014, China. Email: fjzangwd@163.com.
}

Background: The technical safety and efficacy of single-incision plus one-port laparoscopic surgery $(\mathrm{SILS}+1)$ for distal gastric cancer remain unclear. This study was performed to compare the short-term outcomes of patients with distal gastric cancer undergoing SILS+1 versus conventional laparoscopic surgery (CLS).

Methods: This randomized controlled trial involved patients with clinical stage cT1b-3N0-2M0 distal gastric cancer. The patients were randomized to the CLS group or SILS+1 group. The surgical and pathologic outcomes, postoperative mortality, and pain intensity were compared between the two groups.

Results: From January 2019 to April 2021, 117 patients were enrolled and assigned to either the CLS group ( $\mathrm{n}=59)$ or SILS+1 group $(\mathrm{n}=58)$. The clinical characteristics, including blood loss, tumor diameter, pathologic stage, number of lymph nodes harvested, and postoperative recovery, were similar between the two groups $(\mathrm{P}>0.05)$. There were no statistically significant differences in the overall postoperative complication rates between the CLS group and SILS+1 group (10.2\% vs. $6.9 \%$, respectively; $\mathrm{P}=0.743)$. The Clavien-Dindo classification was also comparable $(\mathrm{P}=0.435)$. However, the operating time was significantly longer in the CLS group than SILS+1 group (207.0 441.2 vs. $185.1 \pm 40.7 \mathrm{~min}$, respectively; $\mathrm{P}=0.005)$, and the total incision length was significantly shorter in the SILS+1 group than CLS group $(6.1 \pm 0.6$ vs. $7.6 \pm 0.7 \mathrm{~cm}$, respectively; $\mathrm{P}=0.000)$. Moreover, on the third day after surgery, the visual analog scale (VAS) score was significantly higher in the CLS group than SILS+1 group (1.6 \pm 0.6 vs. $0.6 \pm 0.7$, respectively; $\mathrm{P}=0.000$ ).

Conclusions: The results of this study suggest that SILS+1 for distal gastric cancer performed by an experienced surgeon might be a feasible and safe technique with better cosmetic results and less pain in strictly selected patients.

Trial Registration: This trial was registered at www.chictr.org.cn (ChiCTR2100051491).

Keywords: Single-incision plus one-port; gastric cancer; laparoscopic surgery; gastrectomy

Submitted Sep 11, 2021. Accepted for publication Jan 07, 2022.

doi: $10.21037 /$ tcr-21-1916

View this article at: https://dx.doi.org/10.21037/tcr-21-1916 


\section{Introduction}

Gastric cancer is the fifth most common cancer and the third leading cause of cancer-related deaths among all malignant tumors worldwide (1). Surgical resection is the primary means of treatment for gastric cancer. Compared with open surgery, laparoscopic distal gastrectomy has a better minimally invasive result. Its short-term safety and long-term oncologic effect are comparable with those of open surgery; thus, increasingly more surgeons are preferring this procedure to treat early and advanced distal gastric cancer (2-5). Laparoscopic distal gastrectomy usually requires five ports and an additional small incision. Minimally invasive laparoscopic surgery, including singleport laparoscopic surgery, has gradually emerged with the development of minimally invasive techniques. However, some limitations of these new techniques include instrument interference, loss of triangulation, and coaxial viewing $(6,7)$. Although several studies have suggested how to take advantage of new instruments to overcome the difficulties of single-port gastrectomy $(8,9)$, most such instruments cannot be widely used because of their high price and specialized function. This may explain the restricted generalization of newly developed minimally invasive techniques.

In recent years, laparoscopic surgeons have gradually paid more attention to the technique of adding one operation hole based on single-port laparoscopy, a technique termed single-incision plus one-port laparoscopic surgery (SILS+1). This method improves instrument interference and reduces the difficulty of the operation with conventional surgical instruments. Moreover, the drainage tube can be placed through the operation hole, achieving a reasonable balance between the difficulty of surgery and a minimally invasive aesthetic outcome. This technique has been readily promoted in clinical practice. Some studies have demonstrated that SILS +1 is safe and feasible for gastric cancer and has short-term outcomes comparable with those of conventional laparoscopic surgery (CLS) (10-12). However, these studies had some limitations, including small sample sizes and retrospective designs. Therefore, we conducted a randomized controlled trial to compare the short-term outcomes of SILS+1 versus CLS for distal gastric cancer. This study is presented in accordance with the CONSORT reporting checklist (available at https:// tcr.amegroups.com/article/view/10.21037/tcr-21-1916/rc).

\section{Methods}

\section{Study design and patients}

This single-institution, non-inferiority, randomized controlled trial was conducted in Fujian Cancer Hospital from January 2019 to April 2021. The study was conducted in accordance with the Declaration of Helsinki (as revised in 2013). It was approved by the Ethics Committee of Fujian Cancer Hospital (No. K2021-090-01), and informed consent was obtained from all patients.

The inclusion criteria were an age of 18 to 75 years, pathological diagnosis of gastric cancer, clinical diagnosis of cT1b-3N0-2M0 lesions according to the $8^{\text {th }}$ Edition of the AJCC Cancer Staging Manual (measured using abdominal computed tomography), tumor size of $\leq 5 \mathrm{~cm}$, and planned treatment by subtotal gastrectomy. The exclusion criteria were preoperative radiotherapy and chemotherapy, tumor perforation, and severe mental disease. The withdrawal criteria were invasion of adjacent structures or distant metastasis, treatment by total gastrectomy, and requirement for study withdrawal.

\section{Outcome measures}

\section{Primary endpoint}

The primary endpoint was early morbidity, which included intraoperative and postoperative complications within 30 days after surgery. These complications were classified according to the Clavien-Dindo classification (13).

\section{Secondary endpoints}

The secondary endpoints were operative mortality, postoperative recovery, the cosmetic result, pain intensity, 3 -year disease-free survival, and 5-year overall survival.

\section{Randomization and sample size}

After patients who met the study criteria had provided written informed consent, the administrator divided them into the CLS group and SILS+1 group at a 1:1 ratio using computer randomization. This study had a non-inferiority design. Assuming a dropout rate of 10\%, 60 participants were required per group.

\section{Eligibility of surgeons}

All operations were performed by the same surgeon, who 


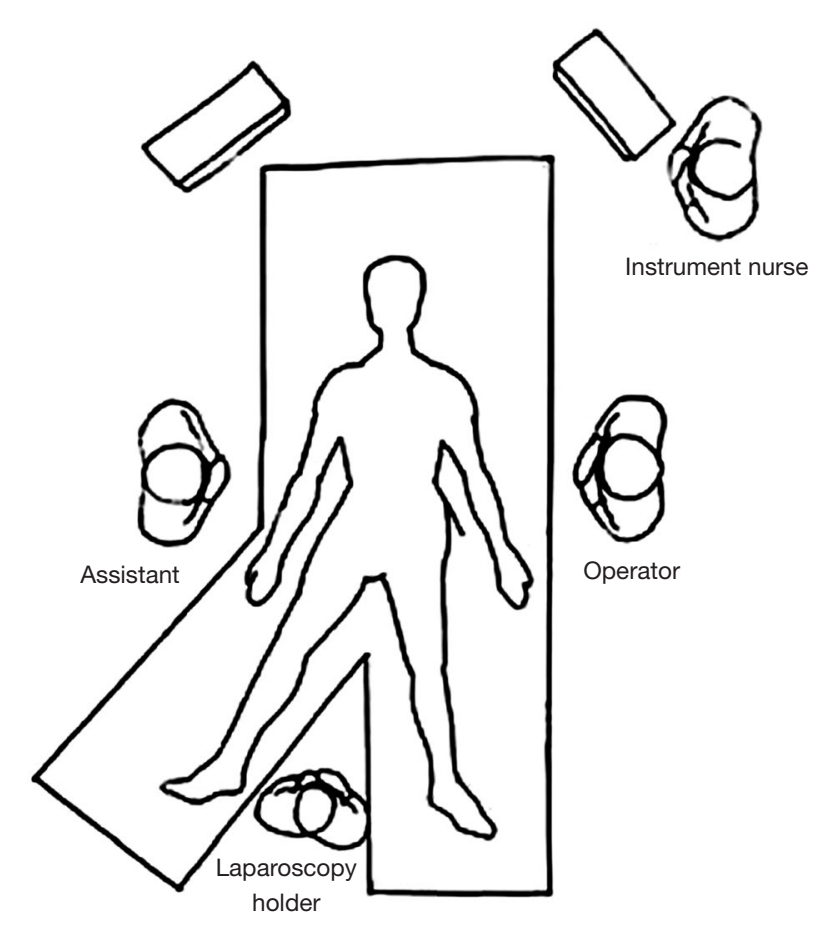

Figure 1 Surgical position.

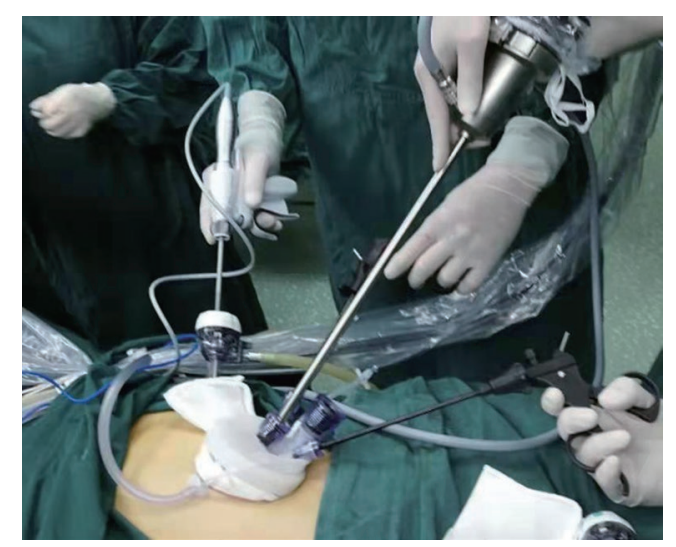

Figure 2 Placement of a 12-mm trocar in the left upper quadrant in the SILS+1 procedure.

had completed more than 300 CLS procedures and 50 SILS+1 procedures.

\section{Surgical technique}

The patients were placed in the supine position with right leg abducted at 45 degrees under general anesthesia. The operator stood on the left side of the patient, and the assistant stood on the right side (Figure 1). In the CLS group, five ports were used to conduct the procedure. A $12-\mathrm{mm}$ trocar was inserted through an umbilical wound, and pneumoperitoneum was established with carbon dioxide. Another $12-\mathrm{mm}$ trocar was inserted in the left preaxillary line $2 \mathrm{~cm}$ below the costal margin as a major hand port, and a 5 -mm trocar was inserted in the left midclavicular line $2 \mathrm{~cm}$ above the umbilicus as an accessory port. Two additional 5 -mm trocars were then placed at the contralateral sites. The intra-abdominal pressure was maintained at 8 to $12 \mathrm{mmHg}$. After abdominal exploration, D2 lymphadenectomy was performed according to the lymph node classification established by the Japanese Gastric Cancer Association (14). The duodenum was transected about $2 \mathrm{~cm}$ distal to the pylorus, and the resection line of the stomach was set at least $5 \mathrm{~cm}$ from the tumor. Both of these procedures were performed using a $45-\mathrm{mm}$ or $60-\mathrm{mm}$ endoscopic linear stapling device. Following these procedures, Billroth II or Roux-en-Y anastomosis was performed with an endoscopic stapling device. The umbilical incision was then extended to a length of 4 to $5 \mathrm{~cm}$, and the specimen was removed.

For the SILS+1 procedure, an initial 3- to $4-\mathrm{cm}$ periumbilical incision was made. A multiport device was then placed at the umbilical incision. A 12 -mm port was used for laparoscope insertion, and the other two ports were used for laparoscopic device insertion. Moreover, a $12-\mathrm{mm}$ trocar was placed in the left preaxillary line $2 \mathrm{~cm}$ below the costal margin and served as the surgeon's dominant operating channel (Figure 2). After the specimen was excised, it was removed from the periumbilical incision where the multiport device had initially been placed.

\section{Informed consent process and safety assessment}

A researcher explained all contents of the informed consent form to potential participants, including the objective and methods of the study, potential risks and costs, and other relevant factors. The participants were instructed tore port any complications, adverse events, or related events associated with treatment, and all information was recorded in detail. After sufficient consideration, the participants signed the informed consent forms.

\section{Statistical analysis}

The statistical analysis was performed using SPSS version 22.0 (IBM Corp., Armonk, NY, USA). Categorical variables are expressed as mean \pm standard deviation and were analyzed using the $\chi^{2}$ test or Fisher's exact test. Continuous 


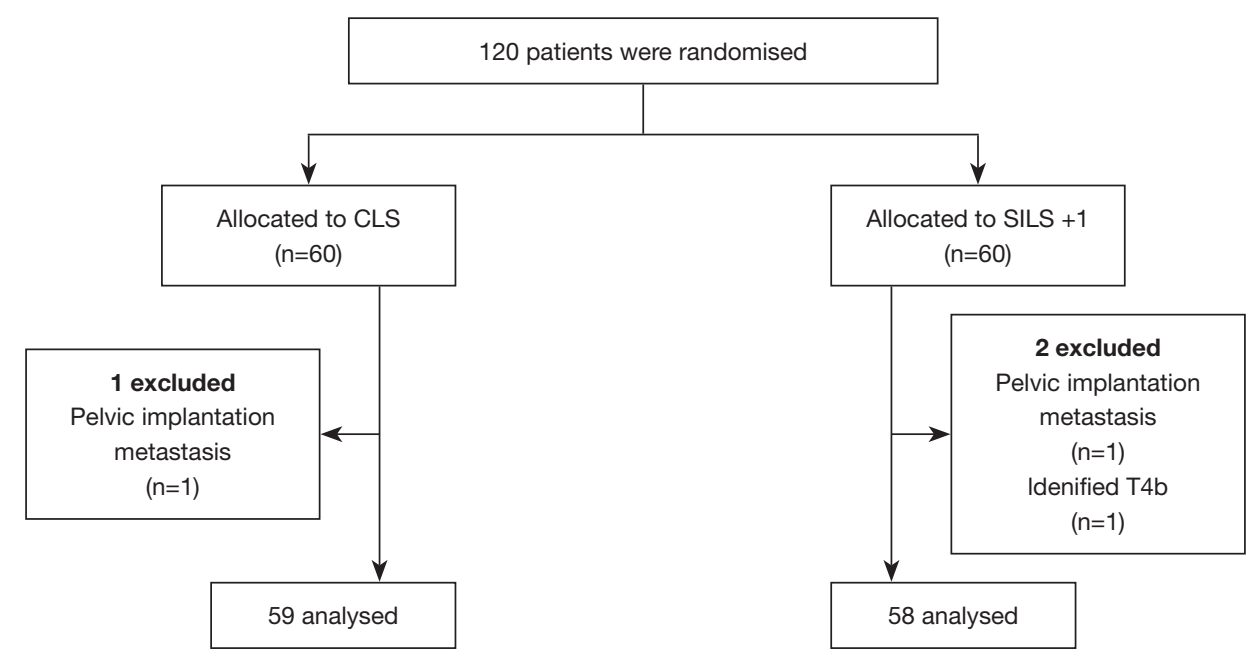

Figure 3 CONSORT diagram. CLS, conventional laparoscopic surgery; SILS+1, single-incision plus one-port laparoscopic surgery.

Table 1 Baseline clinical characteristics

\begin{tabular}{lccc}
\hline Characteristics & CLS $(\mathrm{n}=59)$ & SILS+1 $(\mathrm{n}=58)$ & $\mathrm{P}$ \\
\hline Age (years) & $58.2 \pm 11.3$ & $58.5 \pm 11.1$ & 0.906 \\
Gender & & & 0.619 \\
Male & $35(59.3)$ & $37(63.8)$ & \\
Female & $24(40.7)$ & $21(36.2)$ & \\
BMI (kg/m²) & $23.3 \pm 3.5$ & $22.9 \pm 2.8$ & 0.592 \\
ECOG status & & & 0.755 \\
0 & $52(88.1)$ & $50(86.2)$ & \\
1 & $7(11.9)$ & $8(13.8)$ & \\
ASA grade & & & \\
I & $50(84.7)$ & $47(81.0)$ & \\
II-III & $9(15.3)$ & $11(19.0)$ & \\
History of abdominal & $5(8.5)$ & $4(6.9)$ & 1.000 \\
surgery & & & \\
\hline
\end{tabular}

Data are presented as mean \pm standard deviation or $\mathrm{n}(\%)$. CLS, conventional laparoscopic surgery; SILS+1, single-incision plus one-port laparoscopic surgery; BMI, body mass index; ECOG, Eastern Cooperative Oncology Group; ASA, American Society of Anesthesiologists.

variables are presented as number and percentage and were analyzed using the independent $t$-test or the Mann-Whitney $\mathrm{U}$ test. A two-sided $\mathrm{P}$ value of $<0.05$ was considered significant.

\section{Results}

\section{Patient recruitment}

In total, 120 patients were enrolled from January 2019 to April 2021, and they were randomly assigned to the SILS+1 group or the CLS group. After 1 patient from the CLS group and 2 patients from the SILS+1 group were excluded, 59 patients in the CLS group and 58 patients in the SILS+1 group were analyzed (Figure 3). The patients' clinicopathologic characteristics are presented in Table 1. The baseline characteristics were balanced between the two groups.

\section{Pathologic and surgical outcomes}

There was no statistically significant difference in the pathologic or oncologic outcomes between the two groups (Table 2). The postoperative recovery outcomes were also similar between the two groups. However, the operating time was significantly longer in the CLS group than SILS+1 group $(207.0 \pm 41.2$ vs. $185.1 \pm 40.7 \mathrm{~min}$, respectively; $\mathrm{P}=0.005)$, and the total incision length was significantly shorter in the SILS+1 group $(\mathrm{P}<0.001)$ (Table 3).

\section{Postoperative complications}

No significant difference was observed in the overall postoperative complication rates between the CLS and SILS+1 groups ( $10.2 \%$ vs. $6.9 \%$, respectively; $\mathrm{P}=0.743)$. 
Table 2 Pathologic and oncologic outcomes

\begin{tabular}{lccc}
\hline $\begin{array}{l}\text { Pathologic and } \\
\text { oncologic outcomes }\end{array}$ & $\begin{array}{c}\text { CLS } \\
(\mathrm{n}=59)\end{array}$ & $\begin{array}{c}\text { SILS+1 } \\
(\mathrm{n}=58)\end{array}$ & $\mathrm{P}$ \\
\hline Tumor diameter & $2.1 \pm 1.0$ & $2.2 \pm 1.0$ & 0.392 \\
Pathologic TNM stage & $30(50.9)$ & $37(63.8)$ & 0.236 \\
I & $14(23.7)$ & $13(22.4)$ & \\
II & $15(25.4)$ & $8(13.8)$ & \\
III & $2(3.4)$ & $2(3.4)$ & \\
Differentiation & $25(42.4)$ & $28(48.3)$ & \\
Good & $32(54.2)$ & $28(48.3)$ & \\
Moderate & & & 0.807 \\
Poor/other & $20(33.9)$ & $25(43.1)$ & \\
Depth of invasion & $13(22.0)$ & $18(31.0)$ & \\
T1 & $26(44.1)$ & $15(25.9)$ & \\
T2 & $33.0 \pm 14.0$ & $31.2 \pm 13.1$ & 0.484 \\
T3-4a & & & \\
Harvested No. of LN & & & \\
\hline
\end{tabular}

Data are presented as mean \pm standard deviation or $\mathrm{n}(\%)$. CLS, conventional laparoscopic surgery; SILS+1, single-incision plus one-port laparoscopic surgery; TNM, tumor-node-metastasis; LN, lymph node.

Moreover, the Clavien-Dindo classification was similar between the two groups $(\mathrm{P}=0.435)$ (Table 4).

\section{Postoperative pain}

There was no statistically significant difference in the visual analog scale (VAS) score on postoperative days 1 and 2 or the discharge day between them. On postoperative day 3 , however, the VAS score was significantly lower in the SILS+1 group than in the CLS group $(0.6 \pm 0.7$ vs. $1.6 \pm 0.6$, respectively; $\mathrm{P}=0.000$ ) (Figure 4).

\section{Discussion}

In 1969, Clifford Wheeless reported transumbilical laparoscopic tubal ligation, which was declared to be the first single-port laparoscopic surgery. However, this procedure was not widely carried out in the digestive system until 2011, when Omori et al. (15) reported singleport laparoscopic distal gastrectomy for the first time. To reduce the difficulty of the operation and facilitate clinical promotion, a main operating hole of about $12 \mathrm{~mm}$ has
Table 3 Surgical outcomes

\begin{tabular}{lccc}
\hline Surgical outcomes & CLS $(\mathrm{n}=59)$ & SILS+1 $(\mathrm{n}=58)$ & $\mathrm{P}$ \\
\hline Operation (min) & $207.0 \pm 41.2$ & $185.1 \pm 40.7$ & 0.005 \\
Blood loss $(\mathrm{mL})$ & $110.1 \pm 104.8$ & $95.0 \pm 68.2$ & 0.358 \\
$\begin{array}{l}\text { Conversion to open } \\
\text { surgery }\end{array}$ & 0 & 0 & - \\
$\begin{array}{l}\text { Length of incision to } \\
\text { remove }\end{array}$ & $4.9 \pm 0.7$ & $4.9 \pm 0.6$ & 0.705 \\
$\begin{array}{l}\text { Specimen (cm) } \\
\text { Total length of incision (cm) }\end{array}$ & $7.6 \pm 0.7$ & $6.1 \pm 0.6$ & 0.000 \\
$\begin{array}{l}\text { Time to first flatus (h) } \\
\text { Time to first defecation (h) }\end{array}$ & $94.2 \pm 21.1$ & $66.1 \pm 14.9$ & 0.812 \\
Time to first oral intake (h) & $73.3 \pm 21.3$ & $77.9 \pm 15.4$ & 0.307 \\
Time to first ambulation (h) & $23.9 \pm 4.2$ & $24.6 \pm 4.9$ & 0.451 \\
Additional postoperative & $7(11.9)$ & $2(3.4)$ & 0.163 \\
analgesics & & & \\
Length of postoperative & $9.2 \pm 3.2$ & $8.4 \pm 2.7$ & 0.135 \\
hospital stays (days) & & & \\
\hline
\end{tabular}

Data are presented as mean \pm standard deviation or $n$ (\%). CLS, conventional laparoscopic surgery; SILS+1, single-incision plus one-port laparoscopic surgery.

since been added to the left upper abdomen to separate the surgeon's right hand (which is usually the most actively used hand) during surgical operations for patients with distal gastric cancer. The present study demonstrated similar short-term outcomes between SILS+1 and CLS for subtotal gastrectomy.

As the primary endpoint of this study, early morbidity was mainly measured in terms of intraoperative and postoperative complications within 30 days after surgery. Like in some other studies $(16,17)$, we found no significant difference in intraoperative blood loss between the two groups in our study. However, a previous study suggested that the use of more trocars might increase the probability of trocar-related complications such as bleeding, hernia, organ injury, and infection (18). Although this is outside the context of our study, we plan to evaluate such complications after a longer follow-up period has lapsed.

In this study, one patient in the CLS group developed a grade 3 complication (anastomotic leakage) that required endoscopic therapy. However, no grade 3 complications occurred in the SILS+1 group. The difference was not statistically significant; likewise, the overall postoperative complication rates were the same between the two groups, 
Table 4 Postoperative morbidity and mortality

\begin{tabular}{lccc}
\hline $\begin{array}{l}\text { Postoperative morbidity and } \\
\text { mortality }\end{array}$ & $\begin{array}{c}\text { CLS } \\
(\mathrm{n}=59)\end{array}$ & $\begin{array}{c}\text { SILS+1 } \\
(\mathrm{n}=58)\end{array}$ & $\mathrm{P}$ \\
\hline Postoperative complication & $6(10.2)$ & $4(6.9)$ & 0.743 \\
Bleeding & $1(1.7)$ & $1(1.7)$ & \\
Anastomic leakage & $1(1.7)$ & $0(0)$ & \\
Pulmonary infection & $3(5.1)$ & $1(1.7)$ & \\
Abdominal infection & $1(1.7)$ & $1(1.7)$ & \\
Wound infection & $0(0)$ & $1(1.7)$ & \\
Clavien-Dindo classification & & & \\
I & $1(1.7)$ & 0.435 \\
II & $4(6.8)$ & $4(6.9)$ & \\
III & $1(1.7)$ & $0(0)$ & \\
Reoperation & $0(0)$ & $0(0)$ & - \\
Re-admission within 30 days & $1(1.7)$ & $0(0)$ & - \\
of surgery & & & - \\
Mortality within 30 days of & $0(0)$ & $0(0)$ & - \\
\hline
\end{tabular}

Data are presented as $\mathrm{n}(\%)$. CLS, conventional laparoscopic surgery; SILS+1, single-incision plus one-port laparoscopic surgery.

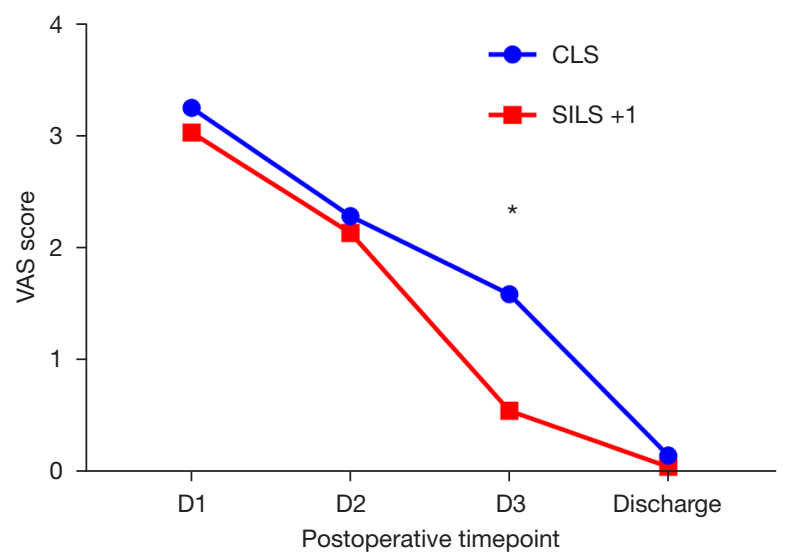

Figure 4 Visual analog scale scores on postoperative days 1 to 3 and discharge day. *, $\mathrm{P}<0.05$. VAS, Visual analog scale.

consistent with previous studies $(10,16,19)$. Moreover, according to existing studies, the overall postoperative morbidity rate of reduced-port or single-port laparoscopic distal gastrectomy ranges from $4.1 \%$ to $42.9 \%$ (20-24). In the present study, the overall postoperative morbidity rate of SILS+1 was $6.9 \%$, which is acceptable. These findings show that SILS +1 is a relatively safe procedure comparable with CLS.

The operating time is an important index with which to evaluate surgical difficulty. Some studies demonstrated that SILS+1 had a similar operating time $(19,25)$, or even a shorter operating time (17). However, Kunisaki et al. (10) retrospectively compared 74 patients who underwent SILS+1 for distal gastric cancer with 74 patients who underwent CLS and found that SILS+1 required longer operating times. Five gastric surgeons participated in that study, and four surgeons had performed fewer than 100 conventional laparoscopic gastrectomies. Inexperience with laparoscopic gastrectomy may have influenced the operating time. In our study, all operations were performed by an experienced surgeon, and the operating time was shorter in the SILS+1 group. The reason for this difference might be that the surgeon independently performed SILS+1 without frequent adjustment by the assistant, which reduced the time spent adjusting and cooperating (26). Furthermore, in contrast to some reports $(10,27)$, we placed the main operating hole in the left upper abdomen instead of on the right side, which could separate the most frequently active hand to reduce interference by the single-hole device. This difference might also reduce the difficulty of surgery and help to shorten the operative time for SILS+1.

Control of postoperative pain is important to reduce patients' discomfort during hospitalization. Pain not only affects patients' physiological state and sleep but also reduces patients' satisfaction and recovery speed (28). Therefore, good pain management is a clinical issue that cannot be ignored. According to previous reports, whether single- or reduced-port laparoscopic surgery can reduce postoperative pain compared with CLS remains controversial. Kim et al. (17) reported that the pain scores on postoperative days 1,3 , and 5 were $3.6,3.2$, and 2.8 in the dual-port totally laparoscopic distal gastrectomy group and $3.7,3.1$, and 2.6 in the conventional surgery group, respectively, with no significant difference between the two groups. However, Liu et al. (29) found that the VAS score at 3 to 5 days after surgery was lower in the reducedport surgery group. In our study, the pain score at 3 days postoperatively was lower in the SILS+1 than CLS group, and the difference was statistically significant. Compared with five-port CLS, SILS+1 involves only three incisions for the auxiliary operation holes; thus, the total incision length is significantly shortened. This may be one of the 
reasons for the lower VAS scores in the SILS+1 group. In addition, most patients began to increase their activity level on the second day after surgery, at which time the difference in pain scores between the two groups became apparent. However, incisional pain might be dependent on various factors, including subjective mood and individual characteristics. More objective indicators and larger samples are required for further evaluation.

Lymph node dissection is one of the most crucial and challenging points in laparoscopic radical gastrectomy. In pure single-port laparoscopic surgery, the difficulty of lymph node dissection is significantly increased because of the lack of an assistant to place force against traction. However, this situation can be effectively improved by adding a main operating hole. In the present study, there was no significant difference in the number of dissected lymph nodes between the two groups. This result indicates that SILS+1 can achieve the same effect as CLS. Kunisaki et al. (30) collected the data of 165 patients undergoing SILS+1 for distal gastric cancer and found that the 5-year survival rate and 5-year disease-specific survival rate were $95.6 \%$ and $98.0 \%$, respectively. In another study that compared patients undergoing SILS+1 versus CLS (10), the 5 -year overall survival rate was $100 \%$ and $96.7 \%$ and the 5 -year disease-free survival rate was $92.3 \%$ and $92.1 \%$, respectively. Both of these results were similar in this study, which further confirms the oncologic efficacy of the SILS+1 technique in the treatment of distal gastric cancer.

The present study has several limitations. All operations were performed by one surgeon in a single center. Although this may have ensured better quality control of the surgical technique, the applicability of this procedure to other surgeons and institutions should be further investigated. Another limitation of this study is that only short-term outcomes were evaluated. As such, the study lacks long-term oncological data. Therefore, we suggest the performance of a multiple-center study in which long-term oncological outcomes regarding recurrence and survival are evaluated.

\section{Conclusions}

For experienced laparoscopic surgeons, SILS +1 is safe and feasible for distal gastric cancer. Moreover, SILS +1 can reduce the operation difficulty compared with pure singleport laparoscopic surgery and has advantages such as a shorter operation time, better cosmetic results, and less pain. Thus, SILS+1 is worthy of clinical promotion.

\section{Acknowledgments}

Funding: This study was sponsored by Fujian Provincial Health Technology Project (Grant No. 2019-2-4); Startup Fund for scientific research, Fujian Medical University (Grant No. 2018QH1223); Bethune Ethicon Excellent Surgery Foundation (Grant No. HZB-20181119-46).

\section{Footnote}

Reporting Checklist: The authors have completed the CONSORT reporting checklist. Available at https://tcr. amegroups.com/article/view/10.21037/tcr-21-1916/rc

Trial Protocol: Available at https://tcr.amegroups.com/article/ view/10.21037/tcr-21-1916/tp

Data Sharing Statement: Available at https://tcr.amegroups. com/article/view/10.21037/tcr-21-1916/dss

Conflicts of Interest: All authors have completed the ICMJE uniform disclosure form (available at https://tcr.amegroups. com/article/view/10.21037/tcr-21-1916/coif). The authors have no conflicts of interest to declare.

Etbical Statement: The authors are accountable for all aspects of the work in ensuring that questions related to the accuracy or integrity of any part of the work are appropriately investigated and resolved. The study was conducted in accordance with the Declaration of Helsinki (as revised in 2013). The study was approved by the Ethics Committee of the Fujian Cancer Hospital (No. K2021-090-01) and informed consent was taken from all the patients.

Open Access Statement: This is an Open Access article distributed in accordance with the Creative Commons Attribution-NonCommercial-NoDerivs 4.0 International License (CC BY-NC-ND 4.0), which permits the noncommercial replication and distribution of the article with the strict proviso that no changes or edits are made and the original work is properly cited (including links to both the formal publication through the relevant DOI and the license). See: https://creativecommons.org/licenses/by-nc-nd/4.0/.

\section{References}

1. Bray F, Ferlay J, Soerjomataram I, et al. Global cancer statistics 2018: GLOBOCAN estimates of incidence and 
mortality worldwide for 36 cancers in 185 countries. CA Cancer J Clin 2018;68:394-424.

2. Hayashi H, Ochiai T, Shimada H, et al. Prospective randomized study of open versus laparoscopy-assisted distal gastrectomy with extraperigastric lymph node dissection for early gastric cancer. Surg Endosc 2005;19:1172-6.

3. Lee JH, Han HS, Lee JH. A prospective randomized study comparing open vs laparoscopy-assisted distal gastrectomy in early gastric cancer: early results. Surg Endosc 2005;19:168-73.

4. Hu Y, Huang C, Sun Y, et al. Morbidity and Mortality of Laparoscopic Versus Open D2 Distal Gastrectomy for Advanced Gastric Cancer: A Randomized Controlled Trial. J Clin Oncol 2016;34:1350-7.

5. Yu J, Huang C, Sun Y, et al. Effect of Laparoscopic vs Open Distal Gastrectomy on 3-Year Disease-Free Survival in Patients With Locally Advanced Gastric Cancer: The CLASS-01 Randomized Clinical Trial. JAMA 2019;321:1983-92.

6. Katsuno G, Fukunaga M, Nagakari K, et al. Short-term and long-term outcomes of single-incision versus multiincision laparoscopic resection for colorectal cancer: a propensity-score-matched analysis of 214 cases. Surg Endosc 2016;30:1317-25.

7. Morales-Conde S, Peeters A, Meyer YM, et al. European association for endoscopic surgery (EAES) consensus statement on single-incision endoscopic surgery. Surg Endosc 2019;33:996-1019.

8. Lee CM, Park DW, Park S, et al. Lymph Node Dissection Using Bipolar Vessel-Sealing Device During Reduced Port Laparoscopic Distal Gastrectomy for Gastric Cancer: Result of a Pilot Study from a Single Institute. J Laparoendosc Adv Surg Tech A 2017;27:1101-8.

9. Kim A, Lee CM, Park S. Is it Beneficial to Utilize an Articulating Instrument in Single-Port Laparoscopic Gastrectomy? J Gastric Cancer 2021;21:38-48.

10. Kunisaki C, Miyamoto H, Sato S, et al. Surgical Outcomes of Reduced-Port Laparoscopic Gastrectomy Versus Conventional Laparoscopic Gastrectomy for Gastric Cancer: A Propensity-Matched Retrospective Cohort Study. Ann Surg Oncol 2018;25:3604-12.

11. Kashiwagi H, Kumagai K, Monma E, et al. Dual-port distal gastrectomy for the early gastric cancer. Surg Endosc 2015;29:1321-6.

12. Zhou $W$, Dong $C Z$, Zang YF, et al. Initial experience of single-incision plus one port left-side approach totally laparoscopic distal gastrectomy with uncut Roux-en-Y reconstruction. World J Gastroenterol 2020;26:4669-79.
13. Dindo D, Demartines N, Clavien PA. Classification of surgical complications: a new proposal with evaluation in a cohort of 6336 patients and results of a survey. Ann Surg 2004;240:205-13.

14. Japanese Gastric Cancer Association. Japanese gastric cancer treatment guidelines 2018 (5th edition). Gastric Cancer 2021;24:1-21.

15. Omori T, Oyama T, Akamatsu H, et al. Transumbilical single-incision laparoscopic distal gastrectomy for early gastric cancer. Surg Endosc 2011;25:2400-4.

16. Kunisaki C, Makino H, Kimura J, et al. Application of reduced-port laparoscopic total gastrectomy in gastric cancer preserving the pancreas and spleen. Gastric Cancer 2015;18:868-75.

17. Kim SM, Ha MH, Seo JE, et al. Comparison of Reduced Port Totally Laparoscopic Distal Gastrectomy (Duet TLDG) and Conventional Laparoscopic-Assisted Distal Gastrectomy. Ann Surg Oncol 2015;22:2567-72.

18. Makino T, Milsom JW, Lee SW. Feasibility and safety of single-incision laparoscopic colectomy: a systematic review. Ann Surg 2012;255:667-76.

19. Yang X, Bu Z, He M, et al. Effectiveness and safety of reduced-port laparoscopic surgery vs conventional multi-port laparoscopic surgery in the treatment of gastric diseases: A meta-analysis. Medicine (Baltimore) 2021;100:e23941.

20. Kim SM, Ha MH, Seo JE, et al. Comparison of single-port and reduced-port totally laparoscopic distal gastrectomy for patients with early gastric cancer. Surg Endosc 2016;30:3950-7.

21. Kim HG, Kim DY, Jeong O. Transition from Conventional to Reduced-Port Laparoscopic Gastrectomy to Treat Gastric Carcinoma: a Single Surgeon's Experience from a Small-Volume Center. J Gastric Cancer 2018;18:172-81.

22. Oh SE, Seo JE, An JY, et al. Compliance with D2 lymph node dissection in reduced-port totally laparoscopic distal gastrectomy in patients with gastric cancer. Sci Rep 2021;11:3658.

23. Lee Y, Kim HH. Single-incision Laparoscopic Gastrectomy for Gastric Cancer. J Gastric Cancer 2017;17:193-203.

24. Lu YM, Lin T, Hu YF, et al. Initial Experience of DualPort Laparoscopic Distal Gastrectomy for Gastric Cancer: A Single-Arm Study. Adv Ther 2019;36:2342-50.

25. Omori T, Fujiwara Y, Moon J, et al. Comparison of SingleIncision and Conventional Multi-Port Laparoscopic Distal Gastrectomy with D2 Lymph Node Dissection for Gastric Cancer: A Propensity Score-Matched Analysis. Ann Surg 
Oncol 2016;23:817-24.

26. Wang Y, Deng H, Mou T, et al. Short-term outcomes of single-incision plus one-port laparoscopic versus conventional laparoscopic surgery for rectosigmoid cancer: a randomized controlled trial. Surg Endosc 2019;33:840-8.

27. Lin T, Mou TY, Hu YF, et al. Reduced Port Laparoscopic Distal Gastrectomy with D2 Lymphadenectomy. Ann Surg Oncol 2018;25:246.

28. Ibrahim MS, Khan MA, Nizam I, et al. Peri-operative interventions producing better functional outcomes and enhanced recovery following total hip and knee

Cite this article as: Teng W, Liu J, Liu W, Jiang J, Chen M, Wei C, Chong CS, Zang W. Comparison of short-term outcomes between single-incision plus one-port laparoscopic surgery and conventional laparoscopic surgery for distal gastric cancer: a randomized controlled trial. Transl Cancer Res 2022;11(2):358-366. doi: 10.21037/tcr-21-1916 arthroplasty: an evidence-based review. BMC Med 2013;11:37.

29. Liu R, Wang Y, Zhang Z, et al. Assessment of treatment options for rectosigmoid cancer: single-incision plus one port laparoscopic surgery, single-incision laparoscopic surgery, and conventional laparoscopic surgery. Surg Endosc 2017;31:2437-50.

30. Kunisaki C, Makino H, Yamaguchi N, et al. Surgical advantages of reduced-port laparoscopic gastrectomy in gastric cancer. Surg Endosc 2016;30:5520-8. 